\title{
Research on College Students' Integrity from the Perspective of Dual-discipline
}

\author{
Zhang Jiongli, Luo Liming, Qin Yajun, Zou Shirao, Ma Huaiyu
}

Civil Aviation Flight University of China, Sichuan Guanghan, 618307

Keywords: dual-disciplinary; integrity of college students; ideological and political education

\begin{abstract}
The dual-disciplinary teaching mode has received widespread attention from major universities, which is conducive to expanding students' horizons and enhancing students' ability. The dual discipline is a teaching model in which different disciplines cross each other and is a derivative of the era of knowledge economy. The society has higher requirements for college students' academic professional knowledge. Interdisciplinary education can help college students to obtain in-depth professional knowledge and comprehensive development of multidiscipline. In the process of carrying out dual-disciplinary teaching, colleges and universities also need to pay attention to the integrity consciousness and behavior of college students, because this is an important part of college students' ideological and political education, and ideological and political education runs through all disciplines. The integrity status of college students affects the overall quality of construction of students, and it is also the focus of quality education. The development of dual-disciplinary teaching also needs to pay attention to the integrity of students to cultivate students' comprehensive quality. The current situation of the integrity of contemporary college students is not optimistic, and there are some problems. In the teaching process of double-disciplinary knowledge, teachers should pay attention to the issue of students' integrity and help students to achieve the "integration of knowledge and action" in order to better promote dual-disciplinary teaching.
\end{abstract}

\section{Introduction}

The dual subject teaching has its own unique teaching advantages, which realizes the innovation and integration of knowledge. The dual-disciplinary teaching can broaden the research horizon of students, promote the exchange and development between different disciplines and realize the borrowing and transformation of disciplinary thinking. Although the dual-disciplinary teaching focuses on the cultivation of students' two different ways of thinking, it has the same ideological and political education tasks as traditional teaching. College students' integrity education is an important part of college students' ideological and political education, and it plays an important role in dual-disciplinary teaching. At this stage, the status of college students' integrity is worrying. The contradiction between college students' honesty awareness and honest behavior is the most prominent problem. In response to the integrity of college students, we need to propose solutions. The article will discuss the current situation of college students' integrity education, and propose corresponding solutions in combination with the dual-disciplinary perspective.

\section{The credibility crisis of college students}

The construction of college students' integrity is the inherent requirement of college students' own development and the requirement to improve the quality of dual-disciplinary teaching. Although most college students have a sense of integrity, there are still some students who have a vague understanding of integrity. At present, the credibility crisis of college students is manifested in the following aspects: the contradiction between integrity awareness and utilitarianism, the conflict between integrity awareness and integrity behavior, and the contradiction in the process of honesty and morality. These credibility crises of college students are reflected in the life, study, job search and other aspects of college students, and are influenced by multiple factors of family, school and society. Then we briefly explain the credibility crisis of college students. 


\subsection{The conflict between integrity awareness and utilitarianism}

The integrity awareness of college students is easily influenced by utilitarianism. Many college students give up the awareness of integrity under the influence of utilitarianism. When college students choose honesty, they will consider their actual interests more, and rarely consider the impact of their behavior on the public interest. Some college students will do things that are against the integrity for their own benefit. This kind of dishonesty may be manifested in violation of one's original intention, or it may be untrustworthy. Observing from the current behavior of college students in school, it is not difficult to find some examples of college students being affected by utilitarianism and losing faith. College students have the phenomenon of cheating in exams because they hope to achieve excellent results in this way, and thus gain an advantage in obtaining scholarships and future employment. Some college students join the student union organization, not to serve classmates, but for the needs of personal self-interest.

\subsection{Conflict between integrity awareness and integrity behavior}

Integrity is a dynamic system consisting of integrity needs, integrity motivation and integrity behavior. According to the theory of moral psychology, integrity behavior is affected by multiple factors, and a person with good sense of integrity does not necessarily have good integrity behavior. College students generally agree with integrity, indicating that college students generally have a strong sense of integrity. The actual situation is that the integrity of college students and the integrity of behavior are not compatible. The integrity behavior of college students is influenced by many factors, including the degree of intimacy of each other, the importance of the event, the integrity of the other one and the interests of the students themselves. Because the integrity behavior of college students is affected by multiple factors, the integrity behavior of college students can easily deviate from the awareness of integrity, thus making things that violate the integrity.

\subsection{Contradictory psychology in the process of integrity and moral identity}

College students' integrity and moral identity are mainly reflected in two aspects. One is the college students' understanding of their own integrity and morality, and the other is the college students' understanding of the integrity of their communities. From the perspective of the current situation of college students' understanding, there is a contradiction between college students' understanding of individual integrity and morality and their moral understanding in communities. Under the influence of college students' unclear understanding of honesty and self-respect, college students have higher evaluation of their own integrity and consider themselves to be honest and trustworthy. For the evaluation of the integrity of their own groups, college students hold the opposite attitude. They believe that the integrity level of their group is not high. This kind of contradictory identity psychology of college students reflects the credibility crisis of college students to a certain extent, indicating that both the dual subject education and the traditional subject education need to strengthen the integrity education for college students.

\section{From the perspective of dual disciplines, the integrity construction of college students need to be strengthened}

The dual subject aims to promote the comprehensive development of students and is conducive to improving students' ability in many aspects. Integrity is an important ability of college students to influence their own development. In the process of dual-disciplinary teaching, teachers should pay attention to the integrity construction of college students and help college students overcome the crisis of integrity. There is also a commonality between the ideological and disciplines, it is not an isolated category. From the perspective of dual disciplines, it is necessary to strengthen the integrity construction of college students. On one hand, teachers should strengthen the integrity education for college students; on the other hand, schools should build a system of college students' integrity and help students establish a sense of integrity. 


\subsection{Innovative forms of college students' integrity education}

The traditional college students' integrity education is mainly carried out through specialized ideological and political courses, which can promote the improvement of college students' integrity consciousness, but they can no longer meet the needs of the development of the times. In the process of dual-disciplinary teaching, teachers can carry out honesty education in a subtle way, integrate integrity education into all aspects of teaching and enable students to enhance their sense of integrity in the process of learning professional knowledge. In addition, teachers should change the traditional one-way teaching and turn to two-way interactive honest teaching, paying more attention to convincing people and affecting people. This kind of honesty education can deepen students' understanding of honesty and realize the internalization of honesty. Teachers' integrity education content should advance with the times and carry out honest education on the hot issues of students' attention.

\subsection{Create a good campus environment}

The impact of the environment on a person is far-reaching and lasting. Colleges and universities should play an active role in the environment for integrity education and create a campus atmosphere of integrity. First of all, through a variety of media, schools can promote the public opinion atmosphere of "honesty and integrity" among students, so that college students can feel the importance and value of integrity. School news media such as school newspapers, radio and television should play an important role in publicity and promote the traditional virtues of honesty and trustworthiness. The school can also carry out some special education activities on integrity, carry out special lectures on honesty, establish a forum with the theme of honesty, and actively guide the integrity construction of college students. Colleges and universities can also integrate the integrity of campus culture with the actual life of students so that students can internalize integrity in the daily practice of learning and life.

\subsection{Establish an integrity incentive system}

A certain incentive mechanism is conducive to the construction of students' integrity and encourages more college students to participate in the construction of integrity. Integrity incentive mechanism is to reward college students with integrity, and impose certain punishments on some college students who violate the integrity. Integrity incentive system needs to record the behavior of students faithfully and resolutely resists some cheating in exams, plagiarism in graduation design and abuse of student loans. The relevant students can be penalized, zero-divided or even cancelled. For some honest students, you can give certain rewards. Schools can promote the deeds of honest students and encourage more students to be honest and trustworthy.

\section{Conclusion}

The dual-disciplinary teaching should not only pay attention to the students' knowledge acquisition, but also cultivate students' ideological and moral qualities. The integrity of college students play an important part of the moral quality of college students and it is also an important indicator to measure students' comprehensive ability. From the perspective of dual-disciplinary, teachers can help college students to build integrity by changing the traditional methods and means of honesty and trust, with the help of honest and trustworthy campus culture. Colleges and universities need to attach importance to the construction of campus integrity culture, carry out corresponding special activities of integrity and establish an incentive system to help college students get rid of the crisis of integrity. Although the integrity of college students is difficult to develop and the effectiveness of the solution is limited, the future students' integrity awareness and behavior will be continuously improved. 


\section{Acknowledgements}

China Civil Aviation Flight Academy (General Program: 2012, XM1005) Research on the Status Quo and Countermeasures of College Students' Honesty in Civil Aviation Specialty

\section{References}

[1] Pan Li, Li Lei. Comprehensively promote the quality education of college students from the perspective of interdisciplinary subjects[J]. Journal of Henan University of Technology (Social Science Edition), 2011, 7(04): 122-124.

[2] Zhang Qiang. Research on the development of moral education in colleges and universities from the perspective of interdisciplinary subjects [D]. Xi'an University of Architecture and Technology, 2013.

[3] Luo Xiushan. Research on the Effectiveness of College Students' Ideological and Political Education from Interdisciplinary Perspective[J]. Jiangsu Higher Education, 2015(01): 127-129 